\title{
Cytokine release from the nasal mucosa and whole blood after experimental exposures to organic dusts
}

\author{
T. Sigsgaard*, E.C. Bonefeld-Jørgensen*, S.K. Kjærgaard*, S. Mamas*, O.F. Pedersen*
}

Cytokine release from the nasal mucosa and whole blood after experimental exposures to organic dusts. T. Sigsgaard, E.C. Bonefeld-Jørgensen, S.K. Kjoegaard, S. Mamas, O.F. Pedersen. (C) ERS Journals Ltd 2000.

ABSTRACT: The aim of this study was to assess the cytokine response after nasal exposure to organic dusts.

In a double blinded, crossover study five garbage workers with occupational airway symptoms and five healthy garbage workers were intranasally exposed to endotoxin (lipopolysaccharide LPS), $\beta$-1,3-D-glucan (GLU), Aspergillus sp., compost or the saline dilute for $15 \mathrm{~min}$. Nasal cavity volume and nasal lavage (NAL) were performed at baseline and $3,6,11 \mathrm{~h}$ postexposure. NAL was analysed with differential cell counts, cysteinyl-leukotrienes, tumour necrosis factor $\alpha$, interleukin (IL)-1 $\beta$, IL-6 and IL-8. A whole blood assay on cytokine-release was performed with LPS and GLU.

NAL cytokines neutrophils, lymphocytes and albumin increased significantly at $6 \mathrm{~h}$ after LPS exposure. GLU induced an increase in albumin and a slight increase in IL$1 \beta$ 6-11 h post exposure. In the WBA a significant increase in all cytokines after exposure to LPS as well as GLU was found. Significantly more cells were seen in NAL of the control group $6 \mathrm{~h}$ post LPS exposure.

In conclusion lipopolysaccharide is the most potent inducer of inflammation in the nasal mucosa whereas compost and $\beta-1,3$-D-glucan only induce minor changes. This reaction to lipopolysaccharide is attenuated in workers with occupational airway symptoms. In whole blood assay, however, $\beta-1,3-D-g l u c a n$ also induces cytokine release, indicating a different protective effect of the nasal mucosa towards lipopolysaccharide and $\beta-1,3-D-g l u c a n$.

Eur Respir J 2000; 16: 140-145.
*Institute of Environmental \& Occupational Medicine, University of Aarhus, Aarhus, Denmark, "Unité de Pharmacologie Cellulaire, Unité Associée Institut Pasteur-INSERM U 485, Paris, France.

Correspondence: T. Sigsgaard, Institute of Environmental and Occupational Medicine, Aarhus University, University Park Building 180, DK 8000 Aarhus, Denmark. Fax: 4589426199

Keywords: $\beta-1,3-\mathrm{D}-$ glucan

cytokine release

individual susceptibility

lipopolysaccharide

nasal challenge

recycling workers

whole blood assay

Received: August 171999

Accepted after revision March 102000

This study has been supported by the Biomed 2 program from the European Commission. Grant No: BMH4-CT96-0105.
During the past 10 yrs a number of cases of respiratory illnesses and dysfunction among employees in the occupational environment of recycling facilities have been reported $[1,2]$. The respiratory effects have been associated with airbone exposures of organic dust containing microbes or to the compounds that such organisms may emit. Candidates are endotoxin from Gram-negative bacteria, lipopolysaccharides (LPS), $\beta$-1,3-D-glucan (GLU) from mould, and allergens in the form of proteins from the micro-organisms or their spores. Previous investigations using cell cultures and dust from a garbage treatment plant show that such dust is cytotoxic and that the active components of the dust may be associated with fungal growth in the dust [3].

Experimental studies have shown that these organic dusts and LPS are able to induce an inflammatory reaction in the airways. The macrophages, involving cytokines and leukotrienes as mediators orchestrate this process [4-7].

Endotoxin (LPS) has been studied intensely in human exposure studies. The acute effect seen after inhalation has been characterized by a neutrophil inflammation in the airways with an influx of polymorphonucleates (PMNs) and macrophages into the airways during the following hours $[8,9]$. Different GLU are present in organic dusts. Their effects after inhalation have been less studied than the effects of LPS, however they do seem to be complex. Inhalation of GLU has been associated with mucus membrane irritation in a recent study by RYLANDER [10]. In this study $6 \mathrm{ng} \cdot \mathrm{m}^{-3}$ was associated with an increased prevalence of symptoms. Studies of guinea pigs inhaling GLU and LPS have shown an attenuation of the LPS response when there is a simultaneous exposure to both [11]. In other respects, a study of guinea pigs immunized with ovalbumin shows that coexposure with GLU abolishes the stimulation effect.

It has been recognized that atopics are more susceptible to the inhalation of LPS [12], and recently it was demonstrated that exposure to allergens caused an influx of LPS-binding protein (LBP) and soluble CD 14 (CD14, the soluble receptor for the LPS-LBP complex) into the lumen of the airways. This increases the activity of inhaled LPS to induce inflammation by an estimated 100fold, leading to a lower no-effect level for allergic asthmatics.

\section{Materials and methods}

From two previous studies of garbage recycling workers five workers who had never had any respiratory symptoms during work at a recycling facility were included in the 
experimental study $[1,2]$. Five workers with at least four of the occupational asthma-like (OAL) symptoms, (wheeze, chest tightness, dyspnoea, bronchial hyperresponsiveness) and peak expiratory flow variability $>20 \%$ on working days induced by this type of work were also included. The latter group had been out of the facilities for at least 8 yrs. The study was approved by the local Ethics Committee and all participants gave written informed consent.

\section{Exposures}

The exposures in this study were $5 \mathrm{~mL}$ aqueous solutions of LPS (endotoxin from Enterobacter agglomerans), GLU, Aspergillus fumigatus and compost, all in a final concentration of $1 \mathrm{mg} \cdot \mathrm{mL}^{-1}$. As a sham exposure $5 \mathrm{~mL}$ of saline was used. The extracts were stored in tubes with an identification code. This code was known only to the person who blinded the samples.

\section{Skin prick test}

A skin-prick test was performed to evaluate immediate allergic reactions to a panel of 10 common inhalant aller-

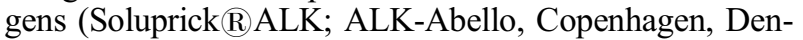
mark).

\section{Pulmonary function testing}

Pulmonary function was tested in accordance with the recommendations of the American Thoracic Society [13]. Predicted values of forced expiratory volume in one second and forced vital capacity were calculated using the standards supplied by the Danish Society of Lung Physicians [14]. The histamine reactivity was determined according to the method described by YAN et al. [15]. The results are reported as provocative dose causing a $20 \%$ fall in FEV1 (PD20) and persons with a PD20 <1.44 mg were considered hyperresponsive.

\section{Acoustic rhinometry}

Measurements of nasal airway dimensions can be performed by methods that apply acoustic reflections from the nasal cavity. Nasal cavity volumes were calculated by integration of the area distance curves from $0-5 \mathrm{~cm}$ (V05), 0-7 cm (V07), and 5-10 cm (V510) into the nasal cavity, respectively. The total nasal volumes V05T, V07T and V510T were calculated as the sum of the two nasal cavities, in order to minimize the bias introduced by the diurnal cycle of the mucosal swelling.

\section{Nasal lavage}

Nasal lavage (NAL) was performed by a bladder catheter with the tip cut off, placed in each nostril and inflated until no air escaped during a forced expiration with closed mouth. After the catheters were secured, the subjects were asked to bend the head forward until the chin reached the chest, whereupon $5 \mathrm{~mL}$ of saline was instilled into each nasal cavity. After $60 \mathrm{~s}$ the cuffing was removed and the liquid was collected in a cup and placed on ice. NALs were centrifuged at $250 \times g$ at $4{ }^{\circ} \mathrm{C}$ for $10 \mathrm{~min}$ and the supernatant was transferred to $1.5 \mathrm{~mL}$ tubes and frozen at $-80^{\circ} \mathrm{C}$.
The pellet was stained with May-Grunwald Giemsa and a total differential count was performed.

\section{Nasal exposure}

The challenge was performed in the same way as the NAL by instillation of $5 \mathrm{~mL}$ of the exposure dilution, warmed to $35^{\circ} \mathrm{C}$, into each nasal cavity. The exposures were left for $15 \mathrm{~min}$ and thereafter the cuffing was removed and the liquid allowed to escape into a cup and discarded.

\section{Challenge sessions}

The study was performed as a randomized double-blinded crossover study with five experimental days, in a controlled environment. Each experimental day was separated by at least 14 days from the previous one. On each day the five people had different exposures. The experiments were performed in a climate chamber, at $21^{\circ} \mathrm{C}$ and $50 \%$ humidity of clean filtered air. Acoustic rhinometry was measured at time $0,30,60,120,180,240,300,360$, 660 and $690 \mathrm{~min}$. NAL was performed at time $0,180,360$ and $660 \mathrm{~min}$.

\section{Cytokine measurements}

The cytokines tumour necrosis factor (TNF)- $\alpha$, interleukin (IL)-1 $\beta$, IL-6 and IL-8 concentrations were determined with a sandwich enzyme linked immunosorbent assay (QUANTIKINE; R\&D, Abingdon, Oxfordshire, UK). Samples were diluted 1:1.25. All samples were tested in duplicate and the mean of two readings is reported here. The coefficient of variation between the two measurements was for all samples $<10 \%$ and the optical density differed $<0.05$ between duplicates.

\section{Cysteinyl leukotriene measurement}

Cysteinyl leukotrienes (LTs) were assayed by enzyme immunoassay (EIA) with use of a commercially available kit (Cayman Chemical Company, Ann Arbor, MI, USA). Acetylcholinesterase from electric eels bound to $\mathrm{LTC}_{4}$ was the enzyme used in the assay. The antibody has the same specificity for $\mathrm{LTC}_{4}$ and $\mathrm{LTD}_{4}$ and shows a cross reactivity at $50 \% \mathrm{~B} / \mathrm{B} 0$ (labelled/unlabelled) of $67 \%$ with $\mathrm{LTE}_{4}$ when tested in a competitive assay. The limit of detectability is $11.8 \mathrm{pg} \cdot \mathrm{mL}^{-1}$. To control the validity of direct $\mathrm{LTC}_{4}$ EIA, different amounts of $\operatorname{LTC}_{4}\left(200,40\right.$ and $\left.20 \mathrm{pg} \cdot \mathrm{mL}^{-1}\right)$ have been added in 10 different lavage samples taken at random. Correlation coefficient was 0.977 and the recovered percentage was $96 \% \pm 25$.

\section{Cytokine release in the whole blood assay}

A blood sample was collected in a pyrogen-free ethylene diamine tetra-acetic acid glass. Each sample was distributed with $0.9 \mathrm{~mL}$ in each of five microfuge tubes, $0.1 \mathrm{~mL}$ of extract in Roswell Park Memorial Institute (RPMI) medium was added, and vortexed. The mixture was left overnight at $37^{\circ} \mathrm{C}$ and then centrifuged for $3 \mathrm{~min}$ at $9,500 \times \mathrm{g}$, in order to collect the supernatant.

The extracts added to the whole blood in this part of the study were aqueous solutions of LPS from Escherichia 
coli, serotype 055:B5 (FLUKA 62 326; Bie \& Berntsen, Copenhagen, Denmark) and GLU (curdlan) from WAKO, Amsterdam, the Netherlands, the final concentrations of the exposures were $10^{4}, 5 \times 10^{3}$, and $1 \mathrm{ng} \cdot \mathrm{mL}^{-1}$ for LPS and $25 \times 10^{4} \mathrm{ng} \cdot \mathrm{mL}^{-1}$ for GLU. As a control $0.1 \mathrm{~mL}$ of RPMI medium was used.

\section{Statistics}

Tabulation, graphical analysis, Mann-Whitney U-test and Wilcoxon test were carried out with SPSS statistical package (SPSS, Inc., Chicago, IL, USA) [16]. For categorized data, the Chi-squared test or Fishers exact tests were performed.

\section{Results}

The majority $(8 / 10)$ of the subjects were smokers five males and five females with more males in the group with earlier symptoms. No person showed any allergy at the skin-prick test, and the lung function measurements were all within normal ranges. Three people showed an increase in histamine responsiveness with a PD20 $<1.44 \mathrm{mg}$ histamine.

The authors found a significant incidence of sinusoidal symptoms after LPS (6/10) compared to GLU (0/10). The difference with regard to other exposures was only borderline significant $(\mathrm{p}=0.057)$, and there was no difference be-
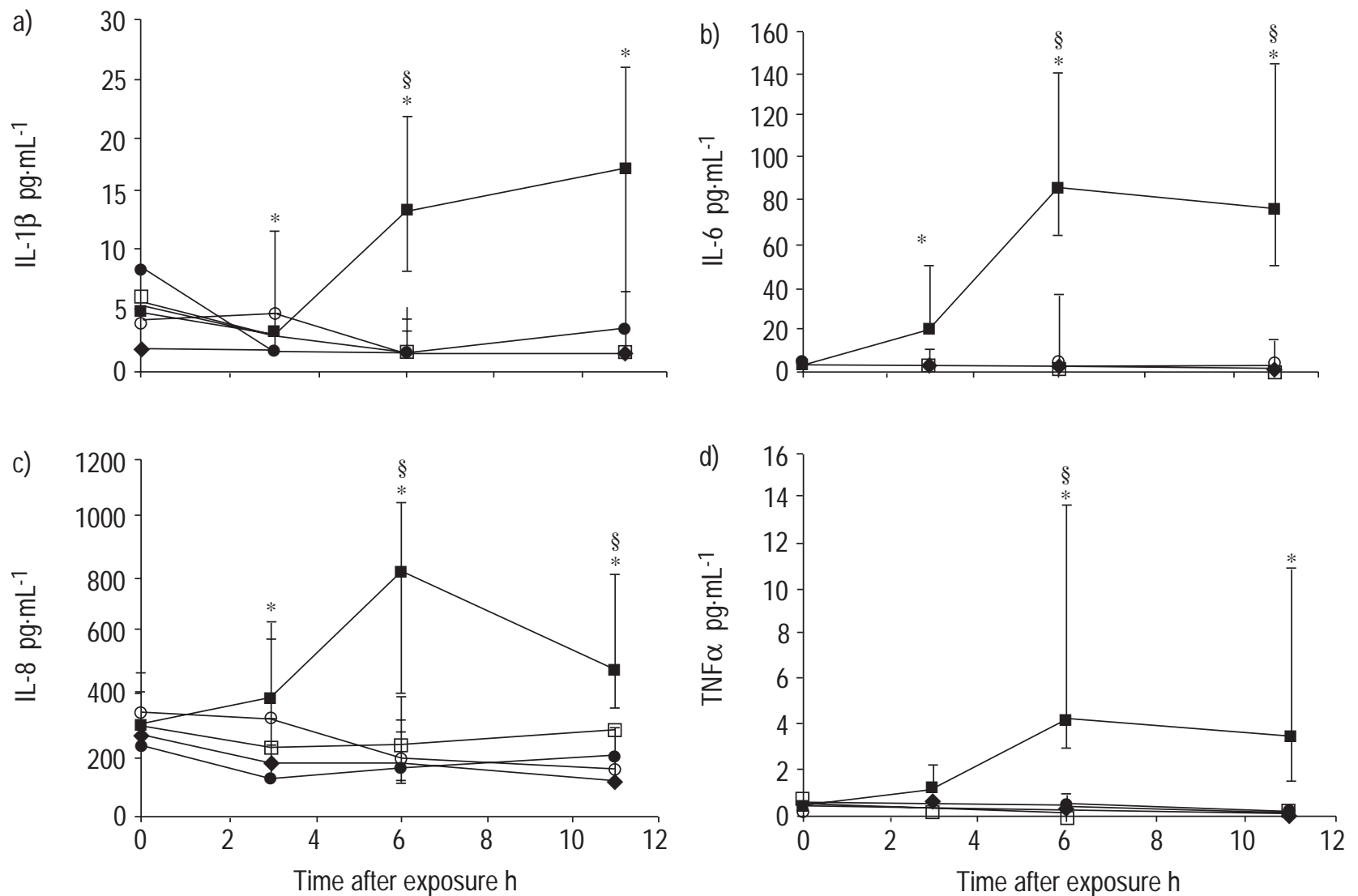

Fig. 1. - a) interleukin (IL)-1 $\beta$, b) IL-6, c) IL-8, d) tumour necrosis factor (TNF) $\alpha$ (median (25-75\% percentiles)) release after exposure, $\bullet$ : saline; lipopolysaccharides (LPS); $: \beta-1,3$-D-glucan; $\square$ : compost; $\bigcirc:$ Aspergillus sp. *:p<0.05 versus baseline (LPS), ${ }^{\S}$ :p $<0.05$ versus 3 h (LPS), Wilcoxon test. tween the occupational asthma group (OAL) and the control group. The most pronounced effect on total nasal volume (TNV) was seen after challenge with GLU where the authors observed an increase of the TNV during the whole session, with a significant increase $6 \mathrm{~h}$ after the challenge.

The cytokine release in the nasal mucosa after the exposures occurred almost entirely after the LPS-exposure with significantly rising IL-6 and IL- 8 after $3 \mathrm{~h}$ and IL-1 $\beta$ plus TNF- $\alpha$ after $6 \mathrm{~h}$. After $6-11 \mathrm{~h}$ all levels of cytokines, except IL-1 $\beta$ started to decline. For all cytokines there was a significant increase in concentration after LPS exposure at $6 \mathrm{~h}$, and for IL- 6 and IL-1 $\beta$ this significant increase was still present at $11 \mathrm{~h}$ postexposure, (fig. 1). $\mathrm{C}_{4}$, urea, uric acid and albumen, was a slight but significant increase in albumen $6 \mathrm{~h}$ post LPS exposure of median (quartiles) $25.1(19.9-41.8) \mu \mathrm{g} \cdot \mathrm{mL}^{-1}$ compared to 8.25 (7.15-10.4) after exposure to saline.

The authors found an increase in lymphocytes and neutrophils after LPS and compost exposure. This increase in cell counts after LPS-exposure was significant compared to all other time-points, however, after compost-exposure this increase was only significant between 3-6 h. Only sporadic eosinophils were found.

When the effects of LPS and GLU were studied in the whole blood assay (WBA), the authors found that both induced a significant release of cytokines, $2-3 \log$ concentrations more than that seen after incubation with the control (fig. 2).
The only effect seen of exposures on the release of LT- 


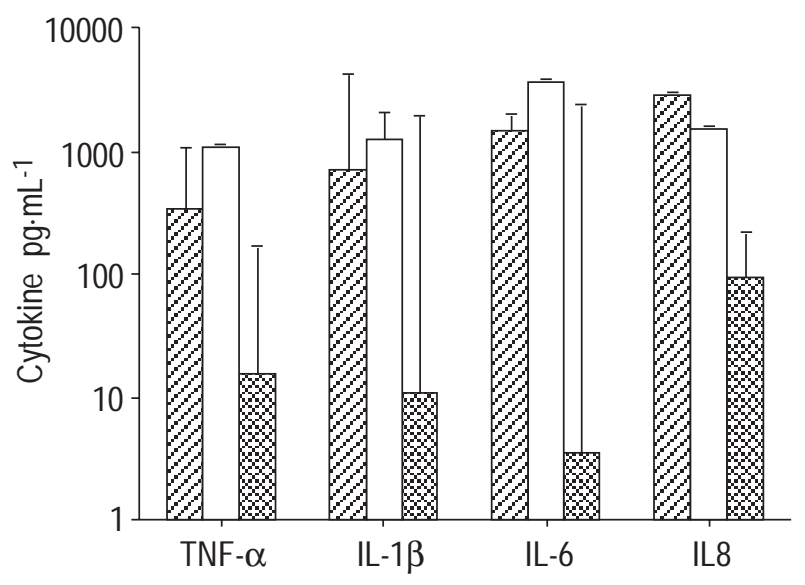

Fig. 2. - Whole blood assay with measurements of tumour necrosis factor (TNF) $\alpha$, interleukin (IL)-1 $\beta$, IL- 6 and IL-8 after $16 \mathrm{~h}$ incubation with lipopolysaccharide (LPS; $\left.10 \mu \mathrm{g} \cdot \mathrm{mL}^{-1} ; \mathscr{Z}\right), \beta-1,3$-D-glucan $(250$ $\mu \mathrm{g} \cdot \mathrm{mL}^{-1} ; \square$ ) and control (网). All groups are significantly different within the same cytokine $\mathrm{p}<0.05$, Wilcoxon test. Data are presented as median and $25-75 \%$ percentiles.

When the authors studied the differences in the effects after exposures stratified for occupational asthma or no occupational asthma, an increase in the cytokine concentration in NAL after exposure to LPS and GLU was found in the OAL-group. This was particularly so at $11 \mathrm{~h}$ postexposure and in the WBA. Only for IL- 8 was a higher response found in the group without OAL. However, none of these differences were significant. The only statistically significant difference between the two groups was a greater increase in PMNs $6 \mathrm{~h}$ postexposure among the people without OAL (fig. 3).

\section{Discussion}

The results of this double-blinded crossover study show that LPS is the most potent inducer of inflammation and symptoms in the nasal exposure system. In the WBA, however, GLU also had an effect on cytokine release. However, exposure to GLU did not lead to symptoms in any of the participants.

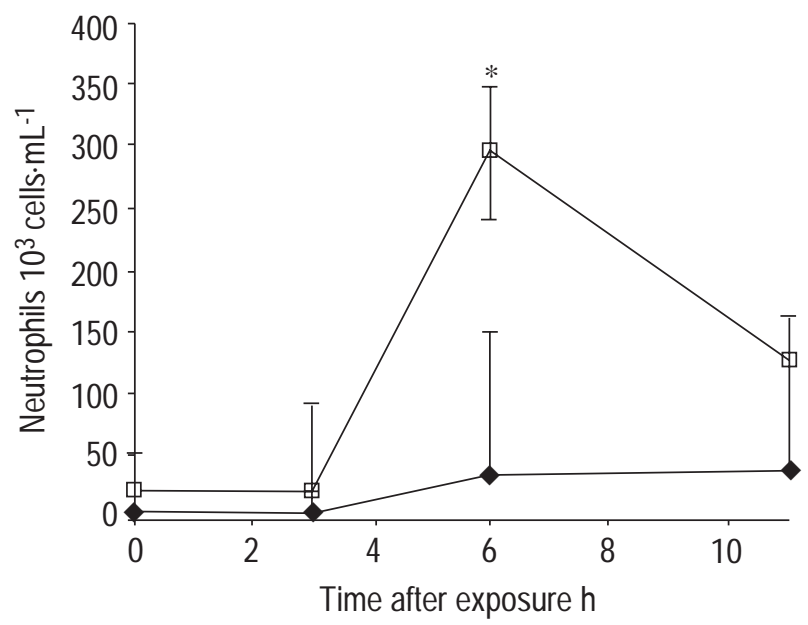

Fig. 3. - The number of neutrophils in nasal lavage after lipopolysaccharide exposure in people with occupational asthma-like symptoms (OAL) $(\diamond)$ and controls $(\square){ }^{*}: \mathrm{p}<0.05$ OAL versus non-OAL, MannWhitney U-test.
The release of cytokines and recruitment of cells to the nasal mucosa seen in this study mimics what has been found in other studies of exposure to LPS or organic material. A recent challenge study with LPS where $5 \mathrm{~mL}(2$ $\left.\mu \mathrm{g} \cdot \mathrm{mL}^{-1}\right)$ was instilled in each nostril for $10 \mathrm{~s}$ showed a peak in IL-6 and IL-8 after $6 \mathrm{~h}$. The levels of these cytokines were much lower, owing to the much lesser exposure [17]. The levels of IL-6 and IL- 8 were 6 and 280 $\mathrm{pg} \cdot \mathrm{mL}^{-1}$ compared to 100 and $800 \mathrm{pg} \cdot \mathrm{mL}^{-1}$ in the present study. The concentration of IL-1 seems to peak later since neither the above-mentioned study nor our study was able to show a decline of IL-1 at 11 or $23 \mathrm{~h}$ postexposure.

Douwes et al. [18] studied the effect of composting work and found an increase in IL-8 concentrations of NAL in composting workers during work-shift. They also found an increased in the number of cells in NAL, and as in the current study they found almost no eosinophils. In contrast to the current study they found an increase in urea whereas the current study, and that by WEBER and DANUSER [17] found an increase in albumen $6 \mathrm{~h}$ post LPS as an indicator of an increased permeability at this point in time. LARSSON et al. [19] found that IL-8 increased from 144 before weighing pigs to $1,064 \mathrm{pg} \cdot \mathrm{mL}^{-1} 6 \mathrm{~h}$ later, which is comparable to what was found in the current study after LPS exposure at $6 \mathrm{~h}$ postexposure. In another study the same group studied IL-1 $\beta$, TNF- $\alpha$ and IL- 6 in NAL and found approximately the same increase in these cytokines $7 \mathrm{~h}$ after weighing pigs as the current authors found $6 \mathrm{~h}$ postexposure [20]. They also, as in the present study, found an influx of neutrophils and lymphocytes postexposure [7]. Exposure to cotton dust has been shown to induce cytokine release in the nasal as well as bronchial mucosa [20] and corn dust extracts also induce a profound increase in neutrophils lasting for $96 \mathrm{~h}$ [22]. Also, TNF- $\alpha$, IL- 6 and IL- 8 levels were sustained for a period of up to $168 \mathrm{~h}$. These are much longer recovery periods in the lungs, compared to what the current study has shown in the nose, probably because the nasal mucosa is more robust and much more readily cleared compared to the lungs. It is interesting to note that the current authors did not find increased numbers of eosinophils in the NAL after any of the exposures, as did Douwes et al. [18]. This is probably because immunoglobulin E mediated inflammation plays a minor role in the reactions seen after these exposures. In the same way, the present study did not show an increase in LTC4, which normally occurs when the leukocyte number increases. The major source is of course the eosinophils. However, it can also be liberated from other stimulated cells like mast cells and macrophages. The concentrations found in the current study are in the same order of magnitude $\left(15-40 \mathrm{pg} \cdot \mathrm{mL}^{-1}\right)$ as what was found in a study of chronic rhinitis patients without allergy [23]. Although the current authors cannot exclude a possible stimulatory effect of instillation on the nasal mucosa which can explain some of the higher $\mathrm{LTC}_{4}$ values [24], this might be an effect of a subchronic inflammation among some of the workers.

Compost was not able to induce increase in cytokine concentrations but a significant increase in neutrophils in NAL was found. The authors included Aspergillus sp. as an exposure since this mould is quite regularly isolated from garbage and compost $[25,26]$ and has been associated with outbreaks of allergic alveolitis [27]. No effects 
of nasal Aspergillus sp. exposure were found on the acute inflammatory response.

It has been postulated that IL- 8 might be a chemoattractant involved in the recruitment of cells to the nasal mucosa. This is partly supported by the current findings since the lymphocytes and neutrophils are absent at $3 \mathrm{~h}$ postexposure when IL- 6 and IL- 8 have increased from baseline. However, after compost exposure the authors found an increase in neutrophils without a preceding increase in cytokines, thus emphasizing the possibility of other mediators of cell-recruitment, or more receptors engaged in the response to these components.

When the authors compared the reactions in people with and without OAL, they found an insignificantly increased response in the OAL-group for most of the parameters studied. For neutrophil recruitment, however, significantly more cells were seen in the NAL of the control group $6 \mathrm{~h}$ post LPS exposure compared to the OAL group, a fact which may reflect a better first line of defence in the former group (fig. 3). Studies of other exposures have shown, that only a proportion of the population is sensitive to exposure to LPS-containing cotton [28]. As the effects seen in this study mainly occurred after LPS and to a minor extent GLU as well as compost exposure it might be expected, that the authors would have found an increased susceptibility among the OAL symptoms persons. This was not the case and therefore it seems that LPS sensitivity is not the sole cause of inflammation in this occupation, or that there is a difference in the reaction in the nose compared to what is seen in the lungs after the same exposure. Finally a subchronic inflammation in the workers would tend to attenuate the differences in reactivity.

The total exposure during each session was $\sim 10 \mathrm{mg}$ of active component. This is a high dose compared to the amount of dust components deposited during a normal working day in the normal inhaled volume of $\sim 4 \mathrm{~m}^{3}$. However, the authors applied the dose topically for only 15 min, allowing the bulk of the exposure to leave the nose again, meaning that the effect should be considerably lower compared to "normal working conditions". Compared to a peak exposure as weighing pigs for $3 \mathrm{~h}$ [19], the authors found comparable cytokine concentrations and cell counts. Hence, it is concluded that the exposures used in this study are relevant for occupational settings with high exposures to dust and LPS.

The authors would ideally only have included nonsmokers in this study. However, as the vast majority of the persons with OAL symptoms were smokers the authors had to match that in the design. To avoid some of the variation all participants were asked not to smoke for the $2 \mathrm{~h}$ prior to the sessions. Still, smoking might have attenuated the response to the instillations, and hence have obscured the differences that the authors were looking for between the people with and without OAL.

This study is small, and the lack of difference between controls and OAL has to be taken with some caution since there were only five people in each group, giving a low power to detect no difference between groups.

In conclusion this study has shown, that lipopolysaccharide is the most potent inducer of inflammation in the nasal mucosa, after topical application of a range of different components of organic dust in concentrations of 1 $\mathrm{mg} \cdot \mathrm{mL}^{-1}$. The inflammation is comparable to what is seen after exposure to swine confinement buildings and grain dust. For $\beta$-1,3-D-glucan and compost only minor changes were seen post exposure, whereas for Aspergillus sp. no effect was found in the study. It is noteworthy that the authors did not find any signs of an immunoglobulin $\mathrm{E}$ mediated allergy, i.e. neither eosinophils nor leukotriene $\mathrm{C}_{4}$ were increased in nasal lavage after exposure. The whole blood assay showed a significant increase in cytokines after incubation with lipopolysaccharide as well as $\beta-1,3-$ D-glucan in contrast to the nasal mucosa. The authors found significantly less polymorphonucleates in nasal lavage from workers with occupational asthma-like symptoms compared to healthy recycling workers after exposure to lipopolysaccharide indicating a possible difference in the first line of defence between the two groups, which is not reflected in the whole blood. However, the study is small, and therefore the findings should be taken with caution.

\section{References}

1. Sigsgaard T, Bach B, Malmros P. Respiratory impairment among workers in a garbage-handling plant. Am $J$ Ind Med 1990; 1: 92-93.

2. Sigsgaard T, Malmros P, Nersting L, Pedersen C. Work related symptoms and lung function measurements among Danish refuse workers. Am Rev Respir Dis 1994; 149: 1407-1412.

3. Roepstorff V, Sigsgaard T. The cytotoxic potential of household waste during composting. Waste Manage Res 1997; 15: 189-196.

4. Burrell R. Immunomodulation by bacterial endotoxin. Crit Rev Microbiol 1990; 17: 189-208.

5. Sandström T, Bjermer L, Rylander R. Lipopolysaccharide (LPS) inhalation in healthy subjects increases neutrophils, lymphocytes and fibronectin levels in bronchoalveolar lavage fluid. Eur Respir J 1992; 5: 992-996.

6. Malmberg P, Larsson KA. Acute exposure to swine dust causes bronchial hyperresponsiveness in healthy subjects. Eur Respir J 1993; 6: 400-404.

7. O'Sullivan S, Dahlén S-E, Larsson KA, et al. Exposure of healthy volunteers to swine house dust increases formation of leucotrienes, prostaglandin D-2 and bronchial responsiveness to methacholine. Thorax 1998; 53: 10411044.

8. Michel O, Duchateau J, Plat G, et al. Blood inflammatory response to inhaled endotoxin in normal subjects. Clin Exp Allergy 1995; 25: 73-79.

9. Michel O, Nagy A-M, Schroven M, et al. Dose-response relationship to inhaled endotoxin in normal subjects. Am J Respir Crit Care Med 1997; 156: 1157-1164.

10. Rylander R. Investigations of the relationship between disease and airborne (1-3)beta-D-glucan in buildings. $\mathrm{Me}$ dia Inflam 1997; 6: 275-277.

11. Fogelmark B, Sjöstrand M, Williams DL, Rylander R. Inhalation toxicity of $(1,3) \beta$-D-Glucan: recent advances. Media Inflam 1997; 6: 263-265.

12. Michel O, Kips J, Duchateau J, et al. Severity of asthma is related to endotoxin in house dust. Am J Respir Crit Care Med 1996; 154: 1641-1646.

13. American Thoracic Society. American Thoracic Society's standardization of spirometry. Am Rev Respir Dis 1995; 152: $1107-1136$.

14. Danish Society of Lung Physicians. Lung function testing; a recommendation. Copenhagen, Denmark, The National Society for Eradication of Lung-Diseases, 1986; $1-16$. 
15. Yan K, Salome C, Woolcock AJ. Rapid method for measurement of bronchial responsiveness. Thorax 1983; 38: 760-765.

16. Norusis MJ/SPSS INC. SPSS/PC+ V2.0 Base manual for the IBM PC/XT/AT and PS/2. Chicago, IL, SPSS Inc., 1988.

17. Weber C, Danuser B. Inflammatory response in nasal lavage to LPS-provocation in normal and allergic subjects. In: International Academy of Indoor Air Sciences, ed. Indoor Air 99. Edinburgh, IAIAS, 1999; pp. 1114-1119.

18. Douwes J, Dubbeld H, van Zwieten L, et al. Work related acute and (sub-) chronic airways inflammation assessed by nasal lavage in compost workers. AAEM 1997; 4: 149152.

19. Larsson BM, Palmberg L, Malmberg PO, Larsson KA. Effect of exposure to swine dust on levels of IL-8 in airway lavage fluid. Thorax 1997; 52: 638-642.

20. Wang Z, Larsson K, Palmberg L, Malmberg P, Larrson P, Larsson L. Inhalation of swine dust induces cytokine release in the upper and lower airways. Eur Respir J 1997; 10: 381-387.

21. Keman S, Jetten M, Douwes J, Borm PJ. Longitudinal changes in inflammatory markers in nasal lavage of cotton workers. Relation to endotoxin exposure and lung function changes. Int Arch Occup Environ Health 1998; 71: 131-137.
22. Deetz DC, Jagielo PJ, Quinn TJ, Thorne PS, Bleuer SA, Schwartz DA. The kinetics of grain dust-induced inflammation of the lower respiratory tract. Am J Respir Crit Care Med 1997; 155: 254-259.

23. Khani J, Campbell A, Enander I, Peterson CGB, Michel F-B, Bousquet J. Indirect evidence of nasal inflammation assessed by titration of inflammatory mediators enumeration of cells in nasal secretions of patients with chronic rhinitis. J Allergy Clin Immunol 1992; 90: 880-889.

24. Ramis I, Serra J, Rosello J, Picado C, Vidal AA. PGE and $\mathrm{PGF}_{2}$ alpha in the nasal lavage fluid from healthy subjects: methodological aspects. Prostaglandins Leucot Essent Fatty Acids 1988; 34: 109-112.

25. Nersting L, Malmros P, Sigsgaard T, Petersen C. Biological health risk associated with resource recovery, sorting of recycle waste and composting. GRANA 1991; 30: 454457.

26. Reiss J. Moulds in containers with biological wastes. Microbiol Res 1995; 150: 93-98.

27. Toopping MD, Scarisbrick DA, Luczynska CM, Clarke EC, Seaton A. Clinical and immunological reactions to Aspergillus niger among workers at a biotechnology plant. Br J Ind Med 1985; 42: 312-318.

28. Castellan RM, Olenchock SA, Kinsley KB, Hankinson JL. Inhaled endotoxin and decreased spirometric values. N Engl J Med 1987; 317: 605-610. 